\title{
Discussion on the model of community management of chronic diseases in cold areas
}

\author{
Rui Jiang ${ }^{1 \dagger}$, Yuquan Zhao ${ }^{2 \dagger}$, Yongchen Wang ${ }^{1 *}$
}

\begin{abstract}
Because of the overbearing low temperature, cold areas increase the morbidity and mortality of chronic non-communicable diseases (chronic diseases) in exposed populations. With the growth of the aging population and the superposition of lifestyle risk factors, the number of people with chronic diseases in cold areas is climbing, and the family and social burdens are rising. These health-threatening circumstances in the cold areas render the general practitioners to face serious challenges and difficulties in the community management of chronic diseases. This paper summarizes the current situation of chronic disease management in cold areas and explores the relevant management models so as to provide a useful reference for regional health construction, graded diagnosis and treatment, and prevention and control of chronic diseases in China.
\end{abstract}

Keywords

cold area; chronic disease; community management; general practitioner

\section{Fund program}

This work is supported by the National Natural Science Foundation of China (72074065), Cultivation Fund for Key Scientific Research Projects of Harbin Medical University, and Harbin Applied Technology and Development Project (2017RAXXJ052).

Received 21 January 2021 accepted 25 March 2021
1 Department of General Practice, the Second Affiliated Hospital of Harbin Medical University, Harbin, 150001, China

${ }^{2}$ Heilongjiang Academy of Traditional Chinese Medicine, Harbin 150001, China

*Corresponding author Yongchen Wang, E-mail: yongchenwang@163.com

${ }^{\dagger}$ Rui Jiang and Yuquan Zhao contributed equally to this work.
Epidemiological investigation shows that cold climate, as one of the main risk factors of chronic diseases, increases the morbidity and mortality of chronic diseases such as circulatory system and respiratory system, and aggravates the health burden[1-3]. The risk and health burden of cold climate have increased over the past decades, and will continue to increase in the future[4]. China has a vast territory, and different regions have distinct geographical climate, temperature, and other factors as well. These regional variations confer the dynamic differences in society, economy, living environment, living customs, medical culture, etc. in China, thereby significant differences in community health service models, particularly between cold areas and the rest of areas. This paper discusses the management of chronic diseases in cold areas from the aspects of climate feature, disease characteristics, lifestyle, and management mode, as well as other pertinent issues.

\section{The Influence of Climatic Characteristics on Diseases in Cold Areas}

The cold areas of China mainly include Northeast, North and Northwest China, which have complex physical geography, dynamic climate, lengthy chilling wintertime with low and even freezing temperatures, large temperature difference, many cold waves, much and deep snow, thick permafrost, and high wind speed and wind-cooling index[5]. Since the recent years, the adverse effects of extreme weather on human health have been well explored and expounded in numerous epidemiological studies, leading to a broad consensus that extreme cold can seriously impact human health[6-8]. However, there have been thus far few related medical research reports on the regional characteristics, disease spectrum characteristics, prevention, and treatment of chronic diseases in cold areas. Hypothermic stimulation in cold areas excites the sympathetic nerve system and increases catecholamine level in the body, leading to vasoconstriction, blood pressure elevation, heart rate acceleration, and even myocardial ischemia and hypoxia. Platelet activation promotes thrombosis and other pathophysiological changes, leading to the development and exacerbation of the underlying chronic diseases[9]. Professor Liu's team collected and analyzed the data records on the daytime temperature and stroke incidence in 16 cities in China and concluded that low-temperature climate is associated with an increase in stroke mortality, mainly in men and the elderly[10]. Professor Raatikka found that in cold regions such as Northern Europe and Finland, there are temperature-related joint and muscle 
pain symptoms in one third of people[11]. It was reported that a drop in temperature increases the incidence of influenza and chronic obstructive pulmonary disease and causes muscle and joint pain[12]. Air pollution arisen from certain heating methods such as coal stove and hot kang (a traditional long platform made of bricks or other forms of fired clay primarily for sleeping used in the northern part of China) in wintertime can cause pathological changes in lung, accelerate atherosclerosis, and changes cardiac autonomic nervous function[13].

\section{Occurrences and Management of Chronic Diseases in Cold Areas}

\subsection{High Incidence of Chronic Diseases in Cold Areas}

The social and natural environmental factors define the regional living environment of the population residing within the region. Residents living in cold areas are often tougher, more indomitable, and have the ability to resist the cold for tenacious survival[14]. Many factors contribute to fat accumulation and great changes in body shape of people in cold areas, such as short sunshine hours and large indoor-outdoor temperature difference, unhealthy living habits (intake of high-fat food, excessive and frequent drinking, high sodium intake, smoking, etc.), and lack of outdoor activities. These problems are deemed to increase the prevalence of chronic diseases and the associated disabilities. Cardiovascular and cerebrovascular diseases, respiratory diseases, metabolic diseases, and osteoarthrosis are of high prevalence, disability, and mortality rate in cold areas. Taking Xinjiang as an example, the prevalence rate of chronic endocrine and metabolic diseases such as diabetes, hypertension and lipid metabolic disorders has been kept increasing year by year[15]. The prevalence rate of metabolic syndrome in Han and Uygur adults in Xinjiang is as high as $30.7 \%$, and the standardized prevalence rate of age composition reaches $28.9 \%[16]$. Therefore, people living in Northeast and North China, where the mean temperature is below $0^{\circ} \mathrm{C}$ during the cold season, are considered to have a greater risk of developing serious hypertension. Furthermore, rural villagers who engage in agricultural labor are habitually in salty diet with high sodium intake, including pickled vegetables, and such a lifestyle can very likely cause and promote hypertension[17]. For example, in Heilongjiang Province, the prevalence rate of hypertension in the middle-aged and elderly is $38.11 \%$. High sodium intake in this area is crucial factor contributing to the high incidence of orthopedic diseases, diabetic complications, and lens opacity[18-19]. Patients with chronic lung diseases in Qinghai tend to have lower partial pressure of oxygen and are more likely to develop severe hypoxemia, respiratory failure, and chronic pulmonary heart disease[20-21]. Chronic diseases not only seriously threaten the life and health of residents, but also bring greater social and economic losses to the cold areas where the economy is underdeveloped[22-23].

\subsection{Current Situation and Problems of Primary Medical and Health Resources in Cold Areas}

China's cold areas are generally geographically remote and sparsely populated, where the economic development is backward with a large income gap behand the cities outside the region and uneven allocation of medical resources. There is insufficient attention paid to chronic diseases prevention in cold areas[24-25]. In the northwest region, due to a large regional span, dry climate, large temperature difference between day and night, and multiethnic gathering, the primary task of the state government is still focusing on the development and improvement of economy, infrastructure, medical care, and education. In the northeast region, the economy based on heavy industry has been trapped in serious recession, and modern industries are emerging slowly and scarcely. The low birth rate and net outflow of young and middle-aged people quickly turn the region into an aging society with a huge proportion of chronic diseases. Yang et al confirmed that, compared with the cities of more advanced economic development, those with lower levels of economic development are more vulnerable to extreme weather, which is manifested by the negative impact of low temperature climate[26]. The "siphon phenomenon" of tertiary general hospitals is particularly obvious in cold areas with a large number of non-urgent and critically ill patients pouring into the higher-level hospitals for medical treatment, resulting in a great waste of medical resources and the emergence of tertiary hospitals. Grass-roots medical service institutions come into a "deserted" scene[27]. The authorities are aware that it is crucial to screen for hypertension and other risk factors that are mostly asymptomatic before causing a serious disease, such as a stroke or heart attack[28]. However, regular and general health check-up mechanisms are still lacking, partially because the costs of medical services provided by public health facilities to individuals are set low by the government but the costs to the medical institutions as service providers are high. In addition, due to the low-level of economic development in cold areas, the job offer package for personnel working in community medical service departments is unattractive, the employment income and benefits are low, and the opportunity for and prospect of personal career development is uncertain. These realities drain general practice professionals away from community medical and healthcare service organizations on one hand and dissipate the trust in general practitioners from residents on the other hand. As such, the allocation of medical resources is imbalanced, and the goal of the diagnosis and treatment of "minor ailments in the community" is difficult to achieve.

\section{General Practice Concept and Characteristics of Community Management}

Healthcare for people with chronic disease is the major challenge 
confronting our primary health system, and general practice can provide good quality care for patients with most high-prevalence long-term conditions. This has been demonstrated in the care for patients with type 2 diabetes[29-30], hypertension[31-32], and chronic musculoskeletal disorders[33]. The primary health care provided by general practice services can yield maximum health benefits to residents[34-35]. General practitioners strive to achieve full coverage of health management for patients with chronic diseases such as cardiovascular and cerebrovascular diseases, hypertension and chronic obstructive pulmonary disease in cold areas and to strengthen screening and early detection of chronic diseases[36]. Community-based chronic disease management takes general practitioners as the main body and community medical and health service departments as the platform and establish health records of the community residents based upon the information collected from the medical and health service resources. Community-based chronic disease management carries out health education, disease surveillance, primordial and primary prevention, and health care for patients with chronic diseases, formulates chronic disease treatment and intervention programs and implements hierarchical management and twoway referrals. The long-term, comprehensive and systematic disease management model, which takes people as the center, family as the unit, community as the scope, and the maintenance and promotion of overall health status as the direction, provides health care for the whole life cycle and a basic guarantee for other specialties to play a better role.

General practice in China began to enter the exploratory stage in the mid-1980s. Over the past 30 years, the government has made great efforts and supports to general practice and issued a series of general practice-related policies have been issued one after another. General practice has undergone drastic changes and stepped onto the stage of rapid development in our country. On the other hand, the community and people have gradually recognized, accepted and embraced general practice. However, there are significant regional differences in the development of general practice in China. The central and eastern regions of Beijing, Shanghai, Guangzhou, Zhejiang, and other central and eastern regions commenced their development of general practice earlier and thus have richer and better experience than the northern part of the west. Some community health service centers have explored and adopted the general practice development models most suitable for their respective local people. The Fang Zhuang Community Health Service Center, led by Professor Wu Hao, has significant advantages in the community-based home-based oldage medical service model incorporated with the intelligent family doctor optimization and cooperation (IFOC) model, and is well accepted and beloved by the elderly with chronic diseases[37]. Hangzhou Sijiqing Community Health Service Center has achieved remarkable results in implementing the accurate family doctor service model[38]. The community health service department is equipped with a large number of general practitioners and excellent general practitioners with strong professional ability to provide timely, effective, safe, economical, convenient and fast medical and health services for community residents [39]. High-quality general practice service plays an irreplaceable role in maintaining and improving the management of chronic diseases.

\section{Application and Advantages of Community Management Model in Cold Areas}

When the general practice team provides long-term and continuous diagnosis and treatment services for community patients with chronic diseases, it not only provides patients with standardized and reasonable drug treatment, but also provides comprehensive and continuous medical care for patients with chronic diseases. General practitioners essentially focus on the needs of patients and cover personalized treatment plans for chronic diseases[40,41].

\subsection{Health Education for Vulnerable Groups}

As the four cornerstones of human health put forward by $\mathrm{WHO}$, "reasonable diet, moderate exercise, quitting smoking and drinking, and psychological balance" are the basic principle of primary prevention[42]. In biomedical research, vulnerable people refer to the group of healthy people who have relatively weak adaptability to natural and social environments, poor ability to resist pathogenic factors and low ability to support themselves because of their genetic and physiological characteristics. Vulnerable individuals generally include women, children, the elderly, and the disabled. Personalized health education for vulnerable people in cold areas can improve their awareness of chronic diseases and self-efficacy and facilitate their effective role transformation from passive acceptance to active participation to improve treatment compliance. Taking full account of the health status, personality, economic conditions and social background of vulnerable people in cold areas, modern information means and care models with combined online and offline resources are used for community diagnosis and treatment services. Moreover, the traditional health management services such as health knowledge lectures, large-scale free clinics and health check-ups for the elderly are also regularly carried out for residents under the jurisdiction. Spring and autumn provide sufficient sunshine and comfortable ambient temperature and offer a good opportunity for guiding chronic patients for their physical exercise, and rehabilitation training. In autumn and winter seasons, people can experience sudden drops in temperature, and thus patients with cardiovascular and cerebrovascular diseases, respiratory diseases, are recommended to wear masks to protect the airway and minimize the stimulation of cold air to blood vessels. Because of the large daily temperature differences and slippery 
roads covered by snow and ice in wintertime, cares need to be provided to vulnerable persons for guiding them how to keep warm and prevent falls. It is highly advocated to instruct residents to adopt humidification measures to maintain indoor air humidity between $50 \%$ and $55 \%$ against the dry air created by the heating system in cold areas. Utilizing humidifiers or planting green plants are recommended to increase the humidity of the living and working environments. General practitioners often cite real cases and real people around them to guide residents to better understand chronic diseases and abandon professional terms by adopting layman language for easy understanding, instead of using the "paternalistic" preaching of specialists. General practitioners are also required to formulate a personalized and comprehensive management plan for residents according to their personal characteristics, social background, living habits and other relevant aspects[43-44].

\subsection{Encourage Patients with Chronic Diseases to Manage Themselves.}

People with chronic diseases often suffer from multiple conditions. General practitioners play an important role in preparing for cold weather and performing health monitoring on patients with chronic diseases. High-salt and high-fat diets are prevalent in cold areas, which is often accompanied by spirits and medicinal wines to warm the body and protect against the cold. Therefore, general practitioners should pay attention to primary prevention and carry out timely and effective intervention on the unhealthy lifestyle and eating habit. It is not easy for individuals to modify their lifestyle by reducing salt intake and refraining from tobacco smoking, because such health risk behaviors are widely adopted by residents ever since their childhood in the communities as a food culture. Community-wide approaches to lifestyle improvement are effective, as demonstrated by Chinese traditional exercise practiced by groups of elderly people in the community[45], and salt-reduction activities in rural Japan[46-47]. Patients with mild hypertension and early diabetes can be instructed to reduce their blood pressure and blood sugar to normal levels through exercise, low-salt and low-fat diet, and weight loss. Long-term home oxygen therapy and respiratory function exercise are important approaches for the treatment of chronic obstructive pulmonary disease. In cold areas, sunshine duration in wintertime is short, diurnal temperature variation is large, and the outdoor activity time for residents is reduced. Under such circumstances, general practitioners can make full use of the information means of "Internet + Medical Care" to carry out online appointment and consultation for chronic diseases, as well as offline medical drug distribution and cloud live health science popularization. Patients with chronic diseases can obtain diagnosis and treatment service suggestions from general practitioners via network video programs at home[48-49], thereby better instructions on home-based exercise or rehabilitation training.
Through systematic and scientific community health education and medical consultation, residents can better understand and aware that chronic diseases are generally lifelong conditions. Education and management are important measures of comprehensive healthcare management, and residents' health behavior and selfmanagement ability are the keys.

\subsection{Advocating Community Home Maintenance Service}

Due to the lower levels of economic development and the limited attractiveness to young people in cold areas, the net population outflow continues to rise, resulting in increasing losses of young adults every year and leaving the aging population in place. The establishment of a long-term and effective community home care model not only can improve the quality of life of elderly patients with chronic diseases, but also has positive practical significance in promoting the development of the old-age security system in China, family harmony and social stability. General practitioners establish personal and family records and achieves of chronically ill patients on basic and general information, living habits, disease history, existing health problems, etc., and provide family doctorsigned services for patients[49]. Standardized management is provided to the revisiting patients with chronic diseases, with face-to-face follow-ups not less than 4 times a year. In fact, the form of service with the family doctor contract system belongs to the outpatient service. At present, such a service is mainly for those who are able to take care of themselves and go out to see a doctor. Community home care offers door-to-door service as the main form of services for patients with chronic diseases, who are disabled, unable to go out, or who are inconvenient to go out because of a cold climate. Chronic patients can make appointments with doctors by phone, WeChat, etc. Contracted general practitioners and nurses provide door-to-door medical services to guide the elderly and their families to familiarize themselves with common medication and basic knowledge of first aid and conduct health check-ups for the elderly, providing pain management, wound care, airway care, rehabilitation training, etc. Regular door-to-door service can not only help patients with chronic diseases make personalized medication plans and feedback, but also alleviate the anxiety and stress levels of the elderly who are living alone and are disabled. Such a service can make patients feel better about themselves and face life and disease positively and optimistically with peace of mind[50-51].

\section{Prospects}

The duties of general practice are to provide long-term and continuous diagnosis and treatment services for patients with chronic diseases in the community, with standardized and reasonable drug treatment, as well as comprehensive and continuous medical cares. Community management emphasizes 
not only on patients' physical health status, but also their psychological state, aiming to achieve holistic medical services taking body and mind in a whole. Another equally important task of general practice is to keep records for patients and their families and background for aiding to uncover and identify the potential pathogenic factors from the family life cycle and facilitate the control and improvement of their heath conditions. With the help of the "Internet +" technology, we can improve the followup mode, expand the outpatient follow-up channels, and extend the online consultation services. In this way, we can facilitate the development of the drug distribution model and the integrated follow-up platform with multidisciplinary cooperation and improve the efficiency, quality, and level of chronic disease management. Community management of chronic diseases is the core issue for China's ongoing medical system reform. Multiple and complex chronic diseases coexist with high incidence and large proportion of residents in cold areas. Strengthening community management can not only meet the basic needs for chronic disease diagnosis and treatment of residents, but also make medical services fair, impartial and accessible. Additionally, it can also integrate the resources from all parties in the medical and health service system and promote the participation of all parties in medical services and other health-related activities. The construction of a primary health care system for chronic diseases in cold areas can offer better health management services to community patients.

\section{Conflict of Interest}

Yongchen Wang is an Editorial Board Member. The article was subject to the journal's standard procedures, with peer review handled independently of this Member and his research groups. The authors declare that they have no other competing interests.

\section{References}

[1] Berko J, Ingram D D, Saha S, et al. Deaths attributed to heat, cold, and other weather events in the United States, 2006-2010. Natl Health Stat Rep, 2014; 76: 1-15.

[2] Song X, Wang S, Hu Y, et al. Impact of ambient temperature on morbidity and mortality: An overview of reviews. Sci Total Environ, 2017; 586: $241-254$

[3] Moghadamnia M T, Ardalan A, Mesdaghinia A, et al. Ambient temperature and cardiovascular mortality: a systematic review and meta-analysis. Peer J, 2017; 5: e3574.

[4] Lee W, Choi H M, Lee J Y, et al. Temporal changes in mortality impacts of heat wave and cold spell in Korea and Japan. Environ Int, 2018; 116: 136-146.

[5] Wang Z J, Wang A P, Yang T, et al. Study on basic strategy of scientific dressing in cold environment. Journal of Preventive Medicine of PLA, 2020; 38(5):7-9.

[6] Zhang $Y$, Wang S, Zhang $X$, et al. Association between moderately cold temperature and mortality in China. Environ Sci Pollut Res Int, 2020; 27(21):26211-26220.

[7] Bobb J F, Peng R D, Bell M L, et al. Heat-related mortalityand adaptation to heat in the United States. Environ Health Perspect, 2014; 122(8):811-816.

[8] Huang C, Barnett A G, Wang X, et al. Effects of extreme temperatures onyears of lifelost for cardiovascular deaths: a time series study in Brisbane, Australia. Circ Cardiovasc Qual Outcomes, 2012; 5(5): 609-614

[9] Yu B, Jin S, Wang C, et al. The association of outdoor temperature with blood pressure, and its influence on future cardio-cerebrovascular disease risk in cold areas. J Hypertens, 2020; 38(6):1080-1089.

[10] Yang, J;Yin, P;Zhou, M, et al.The burden of stroke mortality attributable to cold and hot ambient temperatures: Epidemiological evidence from China. Environ Int.2016;92-93:232-8.

[11] Raatikka Vp, Rytkonenm, Nayha S, et al. Prevalence of coldrelated complaints-symptoms and injuries in the general population: The FINRISK 2002 cold sub-study. Int J Bio-meteorol, 2007,51(5):441-448.

[12] Onozuka D, Hagihara A. Variation in vulnerability to extreme- temperature-related mortality in Japan: A 40-year time-series analysis. Environ Res, 2015; 140: 177-184.

[13] Verrier R L, Mittleman M A, Stone P H. Air pollution: an insidious and pervasive component of cardiac risk. Circulation, 2002, 106(8):890892.

[14] Huang Xiao F. The influence of geographical and cultural factors on the economy of Northeast China. Modern Business, 2020; 13: 78-79.

[15] Wu Shengli, Zhu Yujing, Huang Lijuan, et al. Investigation and analysis of chronic diseases and metabolic syndrome of community residents in Karamay. Xinjiang Medicine, 2017; 47(2): 165-168.

[16] Yang Tian, Ma Yitong, Yang Yining, et al. Epidemiological investigation of metabolic syndrome in adult population of Han nationality and Uygur nationality in Xinjiang. Journal of Xinjiang Medical University, 2011; 34(2):129-132.

[17] Zhang Y, Ma D, Cui R, et al. Facilitators and barriers of adopting healthy lifestyle in rural China: a qualitative analysis through social capital perspectives. Nagoya J Med Sci, 2016; 78: 163-173.

[18] Tan Liming, Chen Lu, long Mingyan, et al. Spatial distribution and influencing factors among middle-aged and elderly patients with hypertension. Preventive Medicine , 2019; 31(3): 236-241, 245.

[19] Liu nianjiao, Kuang hongyu, Zuo jiao, et al. Influence of salt intake on lens opacity in type 2 diabetes mellitus patients in cold region of Northern China. J Clin Pathol Res, 2019; 39(10): 2220-2227.

[20] Li Xiaoqin. Effect of family oxygen therapy on elderly patients with chronic obstructive pulmonary disease in Xining. Journal of Highland Medicine, 2018; 28(1): 30-32.

[21] Cao Chengying, Chen Hong, Wang Shengyuan, et al. Intracranial artery density in patients with chronic obstructive pulmonary disease in plateau region. Journal of the Society of Clinical Neurology, 2017; 30(4):300-302.

[22] Griffifiths F. Primary care research into chronic disease: Qualitative outcomes. Eur J G Pract, 2012.

[23] Strong K, Mathers C, Epping-Jordon J, et al. Preventing chronic disease: a priority for global health. Int J Epidemiol, 2006; 35: 492-494.

[24] Yang G, Kong L, Zhao W, et al. Emergence of chronic non- 
communicable diseases in China. Lancet, 2008; 372: 1697-1705.

[25] Dai B, Zhou J, Mei YJ, et al. Can the new cooperative medical scheme promote rural elders access to health-care services? Geriatr Gerontol Int, 2011; 11: 239-245.

[26] Yang Hui, Han Jianjun, Xu Yanli. General Practice Workforce in China: Development, Challenges and Outlooks. Chinese Journal of General Practice, 2019; 22(19): 2267-2279.

[27] Yang Z, Wang Q, Liu P. Extreme temperature and mortality: evidence from China. Int J Biometeorol, 2019; 63(1): 29-50.

[28] Yip W C M, Hsiao W, Meng Q, et al. Realignment of incentives for health-care providers in China. Lancet, 2010; 375:1120-1130.

[29] Overland J, Mira M, Yue D K. Diabetes management: shared care or shared neglect. Diabetes Res Clin Pract, 1999; 44: 123-128.

[30] Griffin S. Diabetes care in general practice: meta-analysis of randomised control trials. BMJ, 1998; 317: 390-396.

[31] Greenfield S, Rogers W, Mangotich M, et al. Outcomes of patients with hypertension and non-insulin dependent diabetes mellitus treated by different systems and specialities. Results from the medical outcomes study. JAMA, 1995; 274: 1436-1444.

[32] Oakeshott $P$, Kerry $S$, Austin A, et al. Is there a role for nurse-led blood pressure management in primary care? Fam Pract, 2003; 20: 469-473.

[33] Baker R, Sanderson-Mann J, Longworth S, et al. Randomised controlled trial to compare GP-run orthopaedic clinics based in hospital outpatient departments and general practices. Br J Gen Pract, 2005; 55 : 912-917.

[34] Iso $H$, Shimamoto $T$, Naito $Y$, et al. Effects of a longtermhypertension control program on stroke incidence and prevalence in a rural community in northeastern Japan. Stroke, 1998; 29: 1510-1518. [35] Nakagawa H, Miura K. Salt reduction in a population for the prevention of hypertension. Environ Health Prev Med, 2004; 9: 123-129. [36] The Lancet. Chinese doctors are under threat. Lancet, 2010; 376(9742): 657.

[37] Rese A, Balabanova D, Danishevski. Implementing general practice in Russia: getting beyond the first steps. BMJ 2005; 331: 204-207.

[38] C. Heffernan, L. Jones, B. Ritchie K, et al. Local health and social care responses to implementing the national cold weather plan. Journal of Public Health, 2017; 40(3): 461-466
[39] Hao, Wang L, Liu, et al. Exploration of community home care medical service mode based on IFOC model in Fangzhuang community health service center. Chinese Journal of General Practice, 2018; 21(34): 4212-4216.

[40] Jiang M, Zhang L, Lin C, et al. Progress of comprehensive reform of primary health care in China. Chinese General Practice, 2017; 20(22): 2683-2690.

[41] Fung C S, Wong C K, Fong D Y, et al. Having a family doctor was associated with lower utilization of hospital-based health services. BMC Health Serv Res, 2015; 15: 42.

[42] BMC Health Serv Res. Outline of the "Healthy China 2030" Plan of the CPC Central Committee and the State Council, 2015; 15: 42.

[43] Qian Fang, Dai Wenyun, Xie Wenqian. Effect evaluation for family doctor precision contract service in Sijiqing Community Health Service Center. Chinese Journal of general practitioners, 2018; 17(6): 434-436. [44] Dennis S M, Zwar N, Griffiths R, et al. Chronic disease management in primary care: from evidence to policy. Med J Aust, 2008; 188 (8 Suppl): S53-S56.

[45] Reynolds R, Dennis S, Hasan I, et al. A systemalic review of chronic disease management interventions in primary care. BMC Fam Pract, 2018, 19(1): 11.

[46] Zhang B, Qu Ting ting, Shen Shuguang. Research of the primary health care range of basic medical and health institutions based on common and frequently occurring diseases. Chinese Hospital Management, 2016; 36(8): 23-25.

[47] Sandra H van Oostrom, H Susan J Picavet, Simone R de Bruin, et al. Multimorbidity of chronic diseases and health care utilization in general practice. BMC Family Practice, 2014; 15: 61.

[48] Harris M F, Hobbs C, Powell Davies G, et al. Implementation of a SNAP intervention in two divisions of general practice: a feasibility study. Med J Aust, 2005; 183 (10 Suppl): S54-S58.

[49] Greenfield G. Foley K. Majeed A. Rethinking primary gatekeeper role. BMJ, 2016; 354: i4803.

[50] National Health Priority Action Council. National Chronic Disease Strategy. Canberra: Australian Government Department of Health and Ageing, 2006.

[51] Bodenheimer T, Lorig K, Holman $\mathrm{H}$, et al. Patient self-management of chronic disease in primary care. JAMA, 2002; 288: 2469-2475. 